16

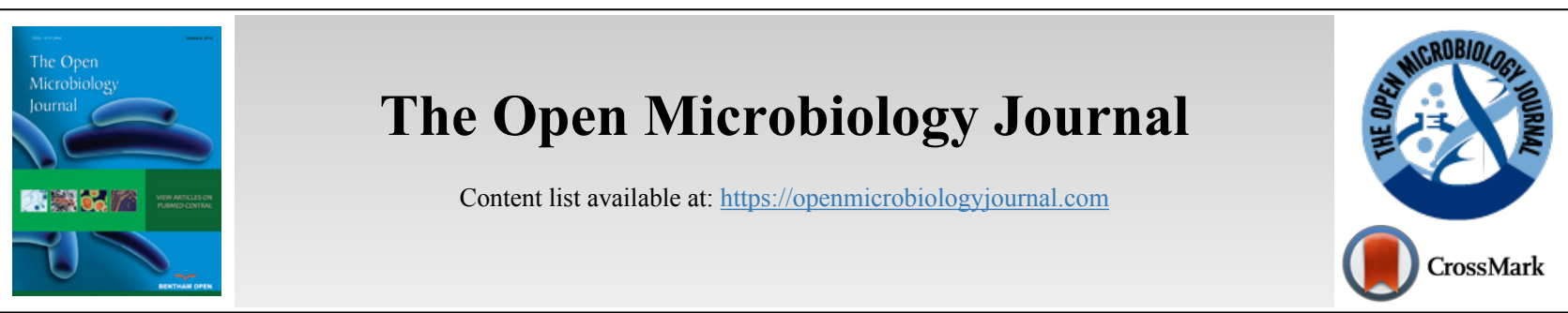

RESEARCH ARTICLE

\title{
Antimicrobial Susceptibility Pattern of $S$. aureus Isolated from Sheep and Goat Carcasses
}

Melaku Tefera ${ }^{1}$, Hailelule Aleme ${ }^{2}$, Samson Girma ${ }^{1, *}$, Abraham Ali ${ }^{1}$, Getachew Gugsa ${ }^{2}$, Firehiwot Abera ${ }^{1}$, Redwan Muzeyin ${ }^{1}$, Tesfaye Legesse ${ }^{1}$, Waktole Gobena ${ }^{1}$, Surafel Fentaw ${ }^{1}$, Rajha Abubaker ${ }^{1}$, Alemayehu Tadesse ${ }^{1}$ and Almaz Gonfa $^{1}$

${ }^{\prime}$ Ethiopia Public Health Institute, Addis Ababa, Ethiopia

${ }^{2}$ Mekele University, Veterinary Medicine, Mekele, Ethiopia

\begin{abstract}
:
Background:

Staphylococcus aureus is a leading cause of food poisoning resulting from the consumption of contaminated food with staphylococcal enterotoxins. Raw meat is a good medium for the survival and spread of drug-resistant $S$. aureus.

Objective:

To look for the prevalence of drug-resistant S. aureus in Addis Ababa abattoir enterprise.

\section{Material \& Methods:}

A cross-sectional study was conducted from November 2013 to April 2014 in Addis Ababa abattoir enterprise. A total of 185 swab samples were collected from the carcasses of sheep, goat and slaughtering materials such as workers' clothes, vehicles, knives and hands. Isolation and identification of $S$. aureus were conducted using the conventional culture methods and signatory tests. Antimicrobial sensitivity was conducted using standard methods.

Results:

The overall prevalence rate for $S$. aureus in the present study was $33 \%$. The higher prevalence rates of $S$. aureus were recorded from sheep carcasses $36 \%$, followed by $30 \%$ from the environment and $16 \%$ from goat carcasses. The variation in the prevalence of $S$. aureus between the carcasses and environment was not statically significant $(p>0.05)$. More than $90 \%$ of $S$. aureus strains were sensitive to vancomycin, chloramphenicol, gentamicin and kanamycin. While $86.9 \%$ S. aureus strains were resistant to penicillin G $80.3 \%$ resistant to ampicillin, $63.9 \%$ resistant to ceftriaxone, $62.3 \%$ resistant to oxacilin, and $62.3 \%$ resistant to cefoxitin respectively.

\section{Conclusion:}

The present study indicated that the quality of slaughtered sheep carcasses was more contaminated by $S$. aureus as compared to goat carcasses, during slaughtering, processing, handling and transportation. The presence of MDR strain in the carcasses demonstrates that there is a growing need to control antimicrobial resistance in sheep and goat carcasses.
\end{abstract}

Keywords: Abattoir, Antimicrobial, Carcasses, Enterotoxin, Sensitivity, S. aureus.

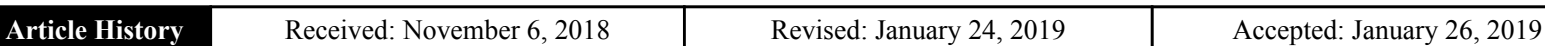

\section{BACKGROUND}

Foodborne diseases often follow the consumption of contaminated food such as meat from infected animals or

\footnotetext{
* Address correspondence to this author at the Ethiopian Public Health Institute, Gulelle, Arbegnoch Street; Tel: +251910485130;

Email: girma.samson@gmail.com
}

carcasses contaminated with pathogenic bacteria such as $S$. aureus. The majority of these microorganisms result from contamination occurring at the slaughterhouse [1], where conventional veterinary inspection cannot detect the presence of these bacteria on apparently healthy looking carcasses [2].

Meat is not only highly susceptible to spoilage, but also 
frequently implicated in the spread of foodborne diseases. Contaminated raw meat is one of the main sources of foodborne diseases, whereby all potentially edible tissues are subjected to contamination from a variety of sources [3].

$S$. aureus is a leading cause of food poisoning resulting from the consumption of contaminated food with staphylococcal enterotoxins [4]. Raw meat is a good medium for $S$. aureus survival and spread of drug-resistant $S$. aureus in the community [5]. The rapid evolution of antibiotic-resistant $S$. aureus is of considerable concern. Multidrug-resistant $S$. aureus strains are especially one of the greatest public concerns since the treatment of infections caused by resistant $S$. aureus is more complicated and difficult [6]. Methicillin-resistant $S$. aureus (MRSA) is known to be one of the most prevalent nosocomial pathogens throughout the world and is capable of causing a wide range of hospital-linked infections [7].

A limited number of investigations have been conducted on the potential of raw meat to spread drug-resistant $S$. aureus as a source of infection. The objective of this study is to look for the prevalence of drug-resistant $S$. aureus in Addis Ababa abattoir enterprise.

\section{MATERIAL AND METHODS}

The study was carried out in Addis Ababa, from November 2013 to April1 2014 at Ethiopian Public Health Institute (EPHI) food microbiology laboratory. Sample collection was carried out at Addis Ababa abattoir enterprise. Analyses of data were performed using IBM SPSS Statistics V21. We considered $p$ values $<0.05$ as statistically significant.

\subsection{Sample Collection}

Sterile cotton swabs moistened in $0.1 \%$ peptone water were used to collect samples. A total of 185 swab samples were collected of which 150 swabs were taken randomly from selected carcasses of sheep, 25 swabs were taken from the carcasses of goats that were slaughtered in Addis Ababa abattoir enterprise. These samples were taken from five different sites viz brisket, shoulder, flank, neck and rump. In addition, 10 samples were taken from the vehicles, workers knives, and their hands at post skinning, evisceration and after washing operations. The collected samples were put in ice container and transported to laboratory examination.

\subsection{Identification of $S$. aureus}

S. aureus was isolated and identified from the samples using conventional culture methods. The transported samples were inoculated into $10 \mathrm{ml}$ of nutrient broth and incubated for 24 to $48 \mathrm{~h}$ at $37^{\circ} \mathrm{C}$. After incubation, the culture was transferred to manitol salt agar plates for isolation of $S$. aureus and incubated for $24-48 \mathrm{hrs}$ at $37^{\circ} \mathrm{C}$. After incubation cultures with typical colonies of yellowish to golden yellow colonies with smooth, slightly raised yellowish colonies were selected for further confirmatory tests. The presumptive positive colonies were plated on blood agar and incubated for $24 \mathrm{hrs}$ at $37^{\circ} \mathrm{C}$. After incubation colonies with alpha and beta hemolysis were screened for gram stain. All the gram-positive staphylococci had passed to tube catalase test, tube coagulase test, and DNase test.

\subsection{Antimicrobial Susceptibility Test (AST)}

AST was carried out on confirmed $S$. aureus using disc diffusion method according to CLSI [8]. Briefly, each of the $S$. aureus isolates was inoculated into $5 \mathrm{ml}$ of trypton soya broth (Oxoid, UK) and incubated at $37^{\circ} \mathrm{C}$ until the suspension turbidity was equivalent to a $0.5 \mathrm{MacFarland}$ standard as measured by turbidity meter before they were tested. The bacterial suspension was subsequently inoculated onto Mueller-Hinton agar plates (Oxoid, UK) and spread over the surface with sterile cotton swabs on a wooden applicator stick.

S. aureus isolates were tested against different classes of antimicrobials, from which the following antibiotics were used. (Penicillins) ampicillin $(30 \mu \mathrm{g})$, penicillin $\mathrm{G}(10 \mu \mathrm{g})$ and oxacillin $(1 \mu \mathrm{g})$, (Macrolides) erythromycin $(15 \mu \mathrm{g})$ and clindamycin $(2 \mu \mathrm{g})$, (cephalosporin) ceftriaxone $(30 \mu \mathrm{g})$, and cefoxitin $(30 \mu \mathrm{g})$ chloramphenicol $(30 \mu \mathrm{g})$ (Aminoglycosides) Kanamycin $(30 \mu \mathrm{g})$ and gentamycin $(10 \mu \mathrm{g})$ (Fluoroquinolones) nalidixic acid $(30 \mu \mathrm{g})$ and (Glycopeptide) vancomycin $(30 \mu \mathrm{g})$ were used.

Antibiotic-impregnated discs were applied to the surface of inoculated plates by hand using sterile forceps. The discs were not closer than $15 \mathrm{~mm}$ to the edge of the plate and they were $24 \mathrm{~mm}$ apart from each other. The plates were incubated inverted aerobically for $18-24$ hours at $37^{\circ} \mathrm{C}$. Finally, the diameters of the zone inhibition around the discs were measured using a transparent ruler according to CLSI guideline [8].

Table 1. Prevalence of $S$. aureus isolated from sheep carcass, goat carcass and the environment.

\begin{tabular}{|c|c|c|}
\hline Sample Source & Number of Samples & S. aureus No (\%) \\
\hline Sheep carcass & 150 & $54(36 \%)$ \\
\hline Goat carcass & 25 & $4(16 \%)$ \\
\hline Environment & 10 & $3(30 \%)$ \\
\hline Total & 185 & $61(33 \%)$ \\
\hline
\end{tabular}

Table 2. The susceptibility pattern of $S$. aureus $(n=61)$ isolated from sheep carcass, goat carcass and environment.

\begin{tabular}{|c|c|c|c|}
\hline Antimicrobials & Susceptible (\%) & Intermediate (\%) & Resistant (\%) \\
\hline Ampicillin & 19.7 & 0 & 80.3 \\
\hline Cefoxitin & 37.7 & 0 & 62.3 \\
\hline
\end{tabular}


(Table 1) contd.....

\begin{tabular}{|c|c|c|c|}
\hline Antimicrobials & Susceptible (\%) & Intermediate (\%) & Resistant (\%) \\
\hline Ceftriaxone & 32.8 & 3.3 & 63.9 \\
\hline Chloramphenicol & 90.2 & 8.2 & 1.6 \\
\hline Clindamycin & 27.9 & 14.8 & 57.4 \\
\hline Erythromycin & 49.2 & 11.5 & 39.3 \\
\hline Gentamicin & 96.7 & 0 & 3.3 \\
\hline Kanamycin & 91.8 & 4.9 & 3.3 \\
\hline Nalidixic acid & 37.7 & 19.7 & 42.6 \\
\hline Oxacillin & 34.4 & 3.3 & 62.3 \\
\hline Penicillin $\mathrm{G}$ & 13.1 & 0 & 86.9 \\
\hline Vancomycin & 100 & 0 & 0 \\
\hline
\end{tabular}

$\chi^{2}=3.921 \mathrm{p}=0.141$

\section{RESULTS}

The overall prevalence of $S$. aureus in sheep, goat carcasses and the environmental samples was $33 \%(61 / 185)$. The highest prevalence of $S$. aureus was found in sheep carcasses $(36 \%)$ followed by swabs from the environment (knife, workers' clothes, slaughtering area and transporting vehi-cles) $30 \%$, and goat carcasses $(16.0 \%)$. No statistically sig-nificant association was found between the sheep and goat carcasses and environmental samples for $S$. aureus contamination $(P>0.05)$ (Table 1).

\subsection{Overall Susceptibility Pattern of $S$. aureus}

The antimicrobials used indicate gentamicin, kanamycin and chloramphenicol were the most effective antibiotics whereby $100 \%, 96.7 \%$ and $91.8 \%$ of the strains were sensitive respectively. Penicillin G, ampicillin, clindamycin and ceftriaxone have showed very poor activity on many isolates, whereby only $13.1 \%, 19.7 \%, 27.9 \%$ and $32.8 \%$ of the isolates were sensitive, respectively. Vancomycin resistance was not observed. Table 2 illustrates the rate of antibiotic resistance among commonly isolated bacteria.

\subsection{Multi-Drug Resistance $S$. aureus}

Multidrug Resistance (MDR) is defined as a single isolate resistant to three or more unique antimicrobial classes. About $21(34.4 \%)$ of the strains were resistant to three or more classes of antibiotics. From which 17(27.8\%), 3(5\%) and 1(1.6\%) of the isolates showed resistance to three, four and five classes of antibiotics respectively. The most frequent multidrug resistance pattern was AMP, CRO, FOX, CHL as shown in Table 3 (Fig. 1).

\section{DISCUSSION}

The study has highlighted that MDR S. aureus occurrence in meat and meat origin represents a threat to public health related to food safety. In the last few decades, staphylococcal food poisoning has been reported as the third cause of foodborne illnesses in the world. Meat, milk and dairy products play a vital role in staphylococcal food poisoning [6].

In the present study, the overall prevalence of $S$. aureus was found to be $33 \%$. This finding is incomparable with the findings observed in Sudan 16\%, [7] 12\% [1] and 32.5 in South Africa from cattle and pig carcasses, respectively [9]. There was no significant difference in bacterial isolates among sheep carcasses, goat carcasses and environment swabs at $P>0.05$. This could be due to the similarity of the hygienic practices between them. In this study all S. aureus isolates were tested for twelve antibacterial agents which showed $86.9 \%, 80.3 \%$, $63.9 \%, 62.3 \%, 62.3 \%, 57 \%, 42.6 \%, 39.3 \%, 3.3 \%, 1.6 \%$ resistance to penicillin $\mathrm{G}$, ampicillin, ceftriaxone, oxacillin, cefoxitin clindamycin, nalidixic acid, erythromycin, gentamicin, kanamycin and chloramphenicol (Table 4).

Table 3. Drug resistance pattern of S.aureus from meat and environmental samples.

\begin{tabular}{|c|c|c|c|c|c|c|}
\hline \multicolumn{6}{|c|}{ Classes of Antibiotics } & \multirow{2}{*}{$\begin{array}{c}\text { Number of Isolates } \\
1 \\
\end{array}$} \\
\hline AMP & CLI & KAN & - & - & - & \\
\hline AMP & CLI & ERY & KAN & & & 1 \\
\hline AMP & $\mathrm{CRO}$ & CLI & ERY & & & 1 \\
\hline AMP & FOX & $\mathrm{CRO}$ & ERY & & & 2 \\
\hline AMP & FOX & $\mathrm{CRO}$ & CLI & & & 10 \\
\hline AMP & FOX & $\mathrm{CRO}$ & CHL & & & 1 \\
\hline AMP & FOX & $\mathrm{CRO}$ & CLI & ERY & & 16 \\
\hline AMP & FOX & $\mathrm{CRO}$ & $\mathrm{CHL}$ & CLI & & 1 \\
\hline AMP & FOX & $\mathrm{CRO}$ & CLI & ERY & KAN & 2 \\
\hline AMP & FOX & $\mathrm{CRO}$ & CLI & ERY & GEN & 2 \\
\hline AMP & FOX & $\mathrm{CRO}$ & CLI & CLI & ERY & 1 \\
\hline
\end{tabular}

AMP Ampicillin, FOX Cefoxitin, CRO Ceftriaxone, CHL Chloramphinicol, CLI Clarithromycin, ERY Erythromycin, KA Kanamycin, GEN Gentamycin. 


\section{Resistant}

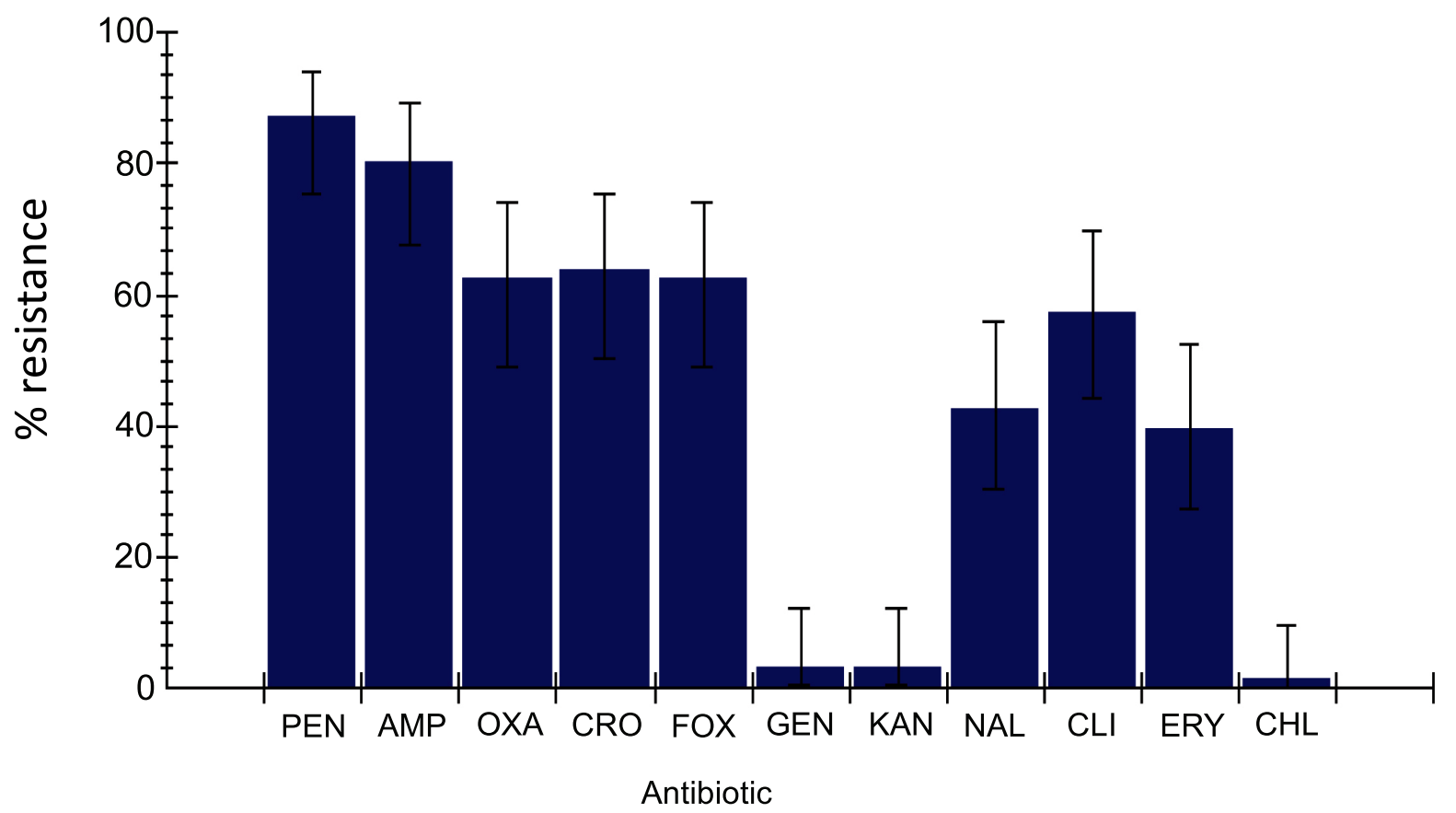

Fig. (1). Percent resistant for S. aureus isolates from carcasses and environments $(n=61)$ for different antibiotics.

Table 4. A summary of multiple drug resistant S.aureus isolates.

\begin{tabular}{|c|c|c|c|}
\hline \multicolumn{2}{|c|}{ Resistance } & Five antibiotics \\
\hline & Three antibiotics & Four antibiotics & 1 \\
\hline Isolates in number & 17 & 3 & 1 \\
\hline
\end{tabular}

In the present study, 21 (34.4\%) S. aureus strain showed multidrug resistance whereby the most common one was resistance primarily to ampicilin, ceftriaxone, clindamycin, cefoxitin. This could be due to the inappropriate use and misuse of $\beta$-lactam and other antibiotics as a means of treatment and thereby the development of resistance. This finding is lower in MDR resistance with the finding in a study [10] which reported $45 \%$ of $S$. aureus strains had resistance to three and more of the tested antimicrobials which is much lower than the study which is $90 \%$ done in Jimma [11]. Based on the result of this study vancomycin, chloramphenicol, gentamicin, and kanamycin were effective against $S$. aureus and this is in agreement with the findings of other investigators [1].

\section{CONCLUSION}

The microbial population and dynamics of foodborne pathogens (S. aureus, Salmonella, Escherichia coli, and others) that comes in contact with fresh meat during slaughtering, dressing and processing present a challenging problem to the meat industry. The condition of the animals prior to slaughter, sanitary condition of the slaughtering environment, and slaughtering materials also contribute to transmission of $S$. aureus through meat and butchers. The occurrence of $S$. aureus in raw meat is of high importance where raw meat is eaten as a cultural food. Moreover, the presence of $75.4 \%$ MDR strain demonstrates the fast-growing strain of $S$. aureus which will become a threat to the public. Thus, it requires a strong and strict implementation of GMP up to the food handlers.

\section{ETHICS APPROVAL AND CONSENT TO PARTI- CIPATE}

Not applicable.

\section{HUMAN AND ANIMAL RIGHTS}

No animals/humans were used for studies that are the basis of this research.

\section{CONSENT FOR PUBLICATION}

Not applicable.

\section{CONFLICT OF INTEREST}

The authors declare no conflict of interest, financial or 
otherwise.

\section{ACKNOWLEDGEMENTS}

I thank all the staff of the public health and clinical microbiology laboratory for their help and collaboration in the development of this study. I also extend my gratitude to Addis Ababa Abattoir staff, who have collaborated and contributed to the collection of samples.

\section{REFERENCES}

[1] Jevons MP. Celbenin-resistant staphylococci. BMJ 1961; 1: 124-5. [http://dx.doi.org/10.1136/bmj.1.5219.124-a]

[2] Brown $\mathrm{MH}$, Gill CO, Hollingsworth $\mathrm{J}$, et al. The role of microbiological testing in systems for assuring the safety of beef. Int $\mathrm{J}$ Food Microbiol 2000; 62(1-2): 7-16.

[http://dx.doi.org/10.1016/S0168-1605(00)00408-6] [PMID: 11139 024]

[3] Bhandare SG, Sherikar AT, Paturkar AM, Waskar VS, Zende RJ. A comparison of microbial contamination on sheep/goat CARCASSES in modern Indian abattoir and traditional meat shops. Food Control 2007; 18: 854-8.

[http://dx.doi.org/10.1016/j.foodcont.2006.04.012]

[4] Tassew H, Abdissa A, Beyene G, Gebre-Selassie S. Microbial flora and food borne pathogens on minced meat and their susceptibility to antimicrobial agents. Ethiop J Health Sci 2010; 20(3): 137-43. [PMID: 22434972]

[5] Chaibenjawong P, Foster SJ. Desiccation tolerance in Staphylococcus aureus . Arch Microbiol 2011; 193(2): 125-35.

[http://dx.doi.org/10.1007/s00203-010-0653-x] [PMID: 21088825]

[6] Ateba C, Mbewe M, Moneoang MS, Bezuidenhout C. Antibiotic resistant Staphylococcus aurous isolated from milk in Mafikeng Area, North West Province, and South Africa. S Afr J Sci 2010; 106: 12-3. [http://dx.doi.org/10.4102/sajs.v106i11/12.243]

[7] Abdalla MA, Siham E, Sluman YY, Alian HA. Microbial contamination of sheep carcasses at modern slaughter house in Khartum state Sud J Vet Sci Anim Husb 2009; 48(1 and 2): 51-6.

[8] Clinical and laboratory standard institute, CLSI Performance standard for antimicrobial susceptibility testing 2017. 27th informational supplement

[9] Scott E, Bloomfield SF. The survival and transfer of microbial contamination via cloths, hands and utensils. J Appl Bacteriol 1990; 68(3): 271-8.

[http://dx.doi.org/10.1111/j.1365-2672.1990.tb02574.x] [PMID: 211 1304]

[10] Abebe M, Daniel A, Yimtubezinash W, Genene T. Identification and antimicrobial susceptibility of $S$. aureus isolated from sheep carcass, goat carcass and isolated from milk samples of dairy cows and nasal swabs of farm workers in selected dairy farms around Addis Ababa, Ethiopia. Afr J Microbiol Res 2013; 7(27): 3501-10.

[11] Fish D, Piscitelli S, Danziger L. A review of antibiotic classes and patient characteristics in 173 studies, pharmacy therapy $1995 ; 15$ : 279-91.

(C) 2019 Jugajyoti et al.

This is an open access article distributed under the terms of the Creative Commons Attribution 4.0 International Public License (CC-BY 4.0), a copy of which is available at: (https://creativecommons.org/licenses/by/4.0/legalcode). This license permits unrestricted use, distribution, and reproduction in any medium, provided the original author and source are credited. 